\title{
Research on Automatic Testing Technology for Parts Looseness of Machining Equipment
}

\author{
Zheng Shuqin \\ Electromechanical Automobile Academy, Changjiang Polytechnic, Wuhan, Hubei, 430074, China \\ email: 987819099@qq.com
}

Keywords: Mechanical Manufacturing, Processing Equipment, Part Loosening, Automatic Detection Technology

\begin{abstract}
Nowadays, the level of science and technology is improving day by day, people attach great importance and attention to the machining of machinery, which also puts forward higher requirements and standards for the precision of machining equipment to a certain extent. In order to further improve the technical level of automatic inspection of mechanical manufacturing equipment parts loosening, it is necessary to carry out comprehensive control and control of the mechanical manufacturing equipment parts loosening. In order to ensure that the inspection mechanism and operation mode are in line with the actual requirements of the automatic inspection system. Therefore, this article mainly uses the mechanical manufacturing equipment parts loose automatic inspection technology research, carries on the following several points analysis, in order to promote the enterprise manufacturing enterprise sustainable development.
\end{abstract}

\section{Introduction}

In order to ensure the quality of mechanical manufacturing equipment parts, it is necessary to carry out the relevant parameter control to ensure that it meets the relevant standards, such as quality and accuracy. At the same time, the technical title provided by the technical personnel cannot be separated from the research on the construction technology of the loose parts of the mechanical manufacturing and processing equipment. The management and operation of modern machinery enterprises need to optimize and improve the automatic inspection technology of mechanical manufacturing equipment parts loosening in order to meet the relevant requirements of the market for mechanical manufacturing processing system and promote the rapid and stable development of machinery manufacturing enterprises.

\section{The Basic Concept, Composition and Characteristics of Detection Technology}

\subsection{Basic Concepts of Detection Techniques}

The use of automatic detection technology in the construction of mechanical manufacturing equipment parts system has a certain guarantee for the accuracy and quality effect of the parts, as shown in figure 1. Testing technology is to adopt all kinds of physical and chemical effects, select the best way, facilities, devices, production, life, scientific research and other related information data to carry out testing, its content to understand, test, analysis, judgment of the technical means [1]. At present, the purchase cost of all kinds of testing technology means is high, but most of the large machinery production enterprises still buy, its purpose is to ensure that the mechanical manufacturing and processing equipment parts meet the relevant quality requirements, and to show the important role of testing technology in the mechanical manufacturing and processing equipment parts. 


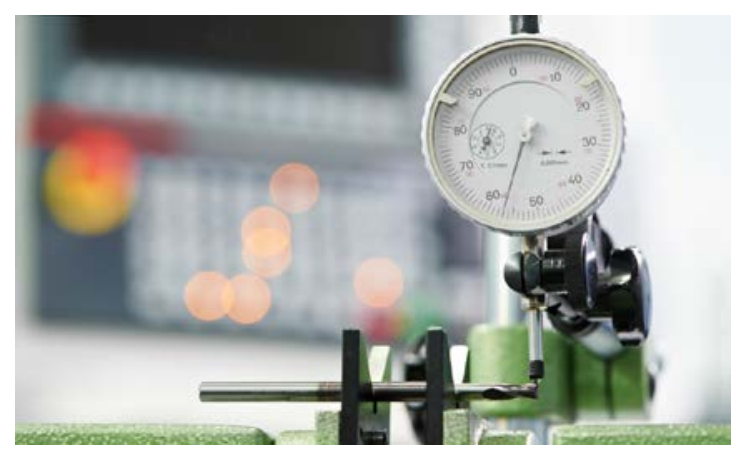

Figure 1 Processing equipment

\subsection{An Overview of the Composition and Characteristics of Detection Techniques}

The detection technology system is mainly composed of automatic signal processing circuit, automatic display recorder, sensor, intermediate converter, automatic protection processing and measurement, calculation and so on. It includes many sensitive elements, output system, measurement elements and various detection device facilities, which have the characteristics of high sensitivity, resolution, linearity, identification force, stability and return error. Effective and accurate test results [2]. In each structure, the sensor, the intermediate converter and the display as the focus component, the mechanical manufacturing equipment parts loose automatic detection technology plays the central role is the sensor, acts as the transmission information carrier, for each kind of information, the data and the energy are detected and transmitted, transmits to the intermediate converter, transmits the effective signal to the display. in order to ensure that the sensor has significant compliance characteristics in a specific period of time, it is necessary to create mathematical models on the basis of detection. In this paper, the design of mathematical model can play the function of data correction, data processing and data analysis, thus reducing the error of information analysis of expression mode. as shown in Figure 2.

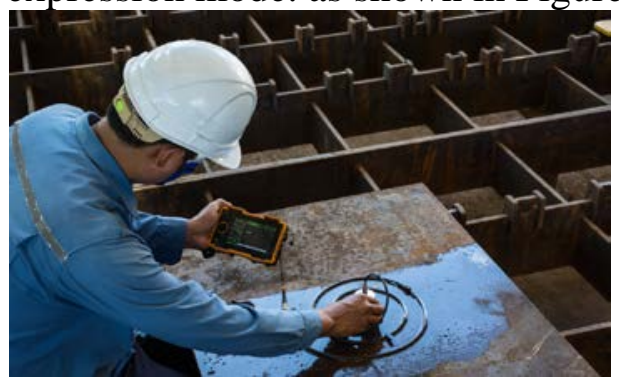

Figure 2 Mathematical model

Testing technology can maximize the compound mechanical manufacturing equipment parts loose automatic detection requirements. On the basis of meeting the requirements of testing, the technical design is mainly simple, economical, easy to operate and maintain, and cannot blindly pursue automation and high standards. The combination of mechanical design and testing technology can further develop the automatic testing technology of machining equipment parts loosening. Therefore, this should be based on the process standards, manufacturing costs, structural complexity, application maintenance, framework complexity, application maintenance convenience and other aspects, to coordinate the processing of mechanical manufacturing equipment parts automation testing technology internal relations [3]. In order to ensure the safety and effectiveness of the overhaul circuit, the following points should be considered comprehensively: first, to ensure the stability and safety of electrical components to meet the basic conditions of the application environment; second, the connection of power supply devices should be consistent with the relevant standards of our country. In a complex automatic detection control circuit, due to the influence of some signals, the circuit will change from one stable state to another, and usually the state of multiple electrical components will change together. Considering the working time of the electrical components, there are several different output states for the sequential circuits, which are also called circuit competition, when the switch circuits, due to the release delay of the electrical components, 
also exist the switching elements are not carried out according to the required logical function output, this phenomenon is called risk.

\section{The Effective Application of Automatic Testing Technology in the Loose Parts System of Machining Equipment}

\subsection{Testing of System Composition}

In order to ensure the normal operation of mechanical manufacturing equipment parts loose system, it is necessary to make it have remarkable stability and reliability characteristics, which requires the relevant technical operators to carry out in-depth study on the system operation structure, operation dimension and other aspects, effectively avoid or find out in time that the mechanical manufacturing equipment parts loose system does not meet the requirements of relevant standards [4]. The use of automated detection technology is not only an effective test of ex post, but also a higher standard for the effectiveness and effectiveness of the test results. During the technical period, the overall mechanical operation dimension, mechanical manufacturing equipment requirements and so on will have a certain impact on the test results, which requires the relevant technical operators to remember the non-contact testing technology or digital testing technology related to the inspection work content, and then promote the mechanical manufacturing equipment parts loose automation construction technology level. During the inspection work, it is possible to use the test results of the relevant technology to ensure its accuracy is effectively guaranteed, such as testing information technology means, fusion technology means and so on, to ensure that the effectiveness of the overall management control system is continuously improved.

In the testing results of loose parts of mechanical manufacturing and processing equipment, centralized management control should be carried out. The main contents are information processing, operation and maintenance. For the detailed division of the test items, the relevant instruments and facilities should be used to obtain accurate results through effective research and analysis of the system. At the same time, it should also be full of information considering the sensitivity, resolution and other parameters of the mechanical manufacturing equipment parts loose automation equipment, as a key factor, on the basis of ensuring the stability of its equipment, the accuracy of the actual measurement value is further improved.

\subsection{Application of Automated Testing Technology in Mechanical Processing Equipment Parts}

At present, in the automatic inspection system of parts loosening of mechanical manufacturing and processing equipment, the main components include sensor device, imaging element device, replacement device and so on, in which the actual function of sensor device can transform the data information, transform the detection data and information into electric signal, and apply relevant technical means to process and integrate it to ensure that the validity of data transmission structure is more prominent, and it can further enhance the data analysis ability of the device, which is of great benefit to the accuracy of data processing results. The function of the replacement device can integrate the information structure and instrument parameters. The final result of the testing needs to be realized by means of imaging components, which also ensures that the technology can meet the standard requirements of the market. Through these three basic components, it can make the automatic detection technology of mechanical manufacturing and processing equipment parts loose to meet the needs of the current automatic production system, and promote the effectiveness and authenticity of the test results.

\section{Effective Application of Testing Technology in Parts System of Machining Equipment}

Nowadays, the level of science and technology in our country is increasing, which promotes the innovation and development of dynamic real-time monitoring technology. Through the efficient use of relevant technology, such as digital flexible coordinate detection, it can guarantee the overall effect of the research work, and apply it to the relevant project construction, which can promote its 
effective play. The efficient application of the relevant technology can ensure the scientific and effective monitoring of the parts loosening of the mechanical manufacturing and processing equipment. In the inspection project, the quantitative qualitative analysis of the parts can be realized automatically and the loosening of the parts can be avoided by using this technical means directly. as shown in Figure 3.

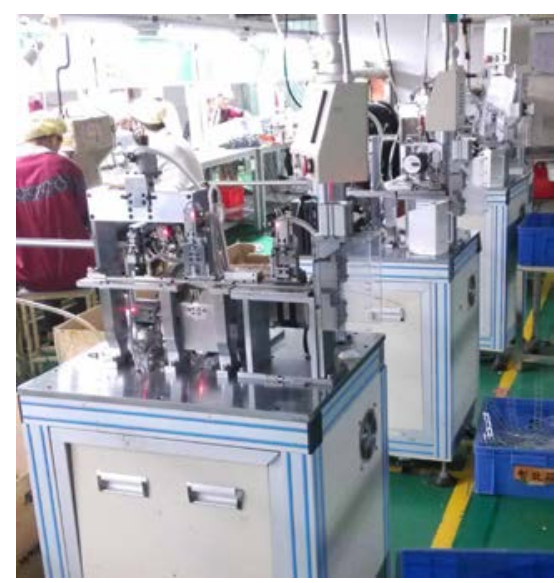

Figure 3 Quantitative qualitative analysis

\section{Conclusion}

To sum up, in view of the effective research on the automatic inspection technology of parts loosening of mechanical manufacturing and processing equipment, the relevant technical operators should carry out the centralized treatment of the problems in the work according to the actual working conditions, and at the same time ensure that the inspection items can be carried out in an orderly manner. In addition, it is necessary to analyze and integrate the automatic inspection data and parameters to improve the accuracy of the operation dimension and system, so as to give full play to the advantage of the automatic inspection technology of parts loosening of machining equipment. Therefore, in the development of modern machinery manufacturing enterprises, it is necessary to combine the detection problem to carry out the systematic and scientific treatment model to promote the rapid and stable development of machinery manufacturing and processing enterprises.

\section{Acknowledgements}

This research has been financed by Hubei skilled personnel training research center project in 2018 "Research on the construction and application of online open courses in Higher Vocational Colleges” (2018JB010).

\section{References}

[1] Liu Yongxun. application of detection technology in automated mechanical manufacturing. Internal Combustion Engine and Accessories, no. 13, pp. 75-76, 2018.

[2] Shen Chunmei. Application of detection technology in automated mechanical manufacturing systems. Electronic World, no. 24, pp. 168-168, 2017.

[3] Wang Suqiong. Research on the Application of Detection Automation Technology in Mechanical Manufacturing Systems. Automation and instrumentation, vol. 15, no. 4, pp. 79-80, 2017.

[4] Zhou Shuyu. Discuss the application of automation technology in machining manufacturing. Internal combustion engine and accessories, no. 15, 2019. 